\title{
Endostatin Is an Independent Risk Factor of Graft Loss after Kidney Transplant
}

\author{
Chang Chu ${ }^{a, b}$ Ahmed A. Hasan ${ }^{c, d}$ Mohamed M.S. Gaballab, e Shufei Zeng ${ }^{a, b}$ \\ Yingquan Xiong ${ }^{b, c}$ Saban Elitok ${ }^{b, f}$ Bernhard K. Krämer ${ }^{b}$ Berthold Hocher ${ }^{b, g, h}$ \\ ${ }^{a}$ Department of Nephrology, Charité, Universitätsmedizin Berlin, Berlin, Germany; ${ }^{\text {b}}$ Fifth Department of Medicine \\ (Nephrology/Endocrinology/Rheumatology), University Medical Centre Mannheim, University of Heidelberg, \\ Heidelberg, Germany; ${ }^{~ I n s t i t u t e ~ o f ~ N u t r i t i o n a l ~ S c i e n c e, ~ U n i v e r s i t y ~ o f ~ P o t s d a m, ~ P o t s d a m, ~ G e r m a n y ; ~}{ }^{\text {d Department }}$ \\ of Biochemistry, Faculty of Pharmacy, Zagazig University, Zagazig, Egypt; ${ }^{\text {e}}$ Faculty of Veterinary Medicine, Benha \\ University, Toukh, Egypt; ${ }^{\mathrm{f} D e p a r t m e n t}$ of Nephrology and Endocrinology/Diabetology, Klinikum Ernst von Bergmann, \\ Potsdam, Germany; ${ }^{9}$ Key Laboratory of Study and Discovery of Small Targeted Molecules of Hunan Province, School of \\ Medicine, Hunan Normal University, Changsha, China; ${ }^{h}$ Institute of Medical Diagnostics, IMD Berlin, Berlin, Germany
}

\section{Keywords}

Endostatin · Graft loss · All-cause mortality · Kidney

transplant recipient

\begin{abstract}
Background: Endostatin is a 20-kDa C-terminal fragment of collagen XVIII, known for its ability to inhibit the proliferation of capillary endothelial cells. Previous studies suggested that circulating endostatin independently predicts incident chronic kidney disease. However, the impact of endostatin on graft loss level in kidney transplant recipients (KTRs) remains unknown. Methods: We conducted a prospective observational cohort study in 574 maintenance KTRs. Patients were followed for kidney graft loss and allcause mortality during a median follow-up of 48 months. Serum-, and urine-samples and clinical data were collected at baseline. Serum Endostatin concentration was analyzed by an ELISA. Results: Among 574 patients, 37 patients had graft loss and 62 patients died. For graft loss, the optimal cut-off value based on receiver operating characteristics analysis (area under the curve $0.79,95 \% \mathrm{Cl} 0.71-0.86, p<$
\end{abstract}

\section{KARGER}

๑) 2020 S. Karger AG, Basel
0.001 ) of endostatin was $147.3 \mathrm{pmol} / \mathrm{L}$. Kaplan-Meier curves revealed that higher serum endostatin concentrations positively correlated with graft loss $(p<0.001)$. Multivariable Cox regression analyses showed that baseline endostatin concentrations were significantly associated with graft loss after adjusting for graft loss risk factors (adjusted hazard ratio [HR] 8.34; 95\% Cl 2.19-31.72; $p=0.002$ ). The adjusted HRs for classical graft loss risk factors such as baseline estimated glomerular filtration rate and urinary protein excretion were lower (1.91 and 5.44, respectively). In contrast to graft loss, baseline endostatin concentrations were not associated with all-cause mortality. Conclusion: Increased serum endostatin at baseline is independently associated with the risk of graft loss in KTRs.

(c) 2020 S. Karger AG, Basel

\section{Introduction}

The global burden of kidney disease is driven by the population growth, aging and increased prevalence of type 2 diabetes [1]. 
Kidney transplantation, if possible, is the preferred way of treatment patients with end-stage kidney disease, because this treatment modality improves both the quality of life and reduces mortality as compared to renal replacement therapy by either hemodialysis or peritoneal dialysis [2-4]. In the 2018 update of the KDIGO Clinical Practice Guideline it is therefore recommended that all patients with advanced chronic kidney disease (CKD), who are expected to reach end-stage kidney disease should be considered for kidney transplantation [5].

Over the past decade, the rate of early graft loss after kidney transplantation was reduced substantially, whereas the rate of chronic graft loss was not significantly improved [6-8]. The underlying mechanism of long-term graft loss is complex and not fully understood. Common risk factors associated with graft loss include alloantigen-dependent factors, such as episodes of acute rejection and human leukocyte antigen mismatching, and alloantigen-independent factors such as tissue injury, inadequate renal mass, and inadequate immunosuppressive therapy [9-11]. The pathology of graft loss is characterized by interstitial fibrosis and tubular atrophy [12].

Endostatin is a $20-\mathrm{kDa}$ C-terminal fragment generated from collagen XVIII, which is a component of the endothelial basement membranes. Endostatin is released through the action of proteases, such as matrix metalloproteinases [13]. Endostatin is known for its ability to inhibit proliferation of capillary endothelial cells [14]. Angiogenesis is the formation of new micro vessels from pre-existing vessels. It is essential for tissue regeneration after ischemic damage, and organ development [15-17]. With respect to kidney function, it was shown that loss of glomerular function is associated with the presence of tubulointerstitial fibrosis, which is characterized by a substantial rarefaction of peritubular capillaries. Dysregulated expression of endogenous angiogenic inhibitors such endostatin might influence the balance between capillary formation and regression, resulting in capillary rarefaction [18-20]. Lin et al. [21] reported that endostatin is increased in aging kidneys, suggesting that elevated endostatin levels are linked to kidney injury in the aging kidneys by promoting renal interstitial fibrosis. Several clinical studies are in line with this hypothesis demonstrating that endostatin is an independent biomarker predicting incident CKD [22-26]. Based on the pre-clinical as well clinical reports of endostatin in CKD, we hypothesized that elevated serum endostatin concentrations might be associated with graft loss, independent of well-established common graft loss risk factors.

\section{Materials and Methods}

We conducted a cross-sectional analysis of a prevalent cohort in 574 prevalent kidney transplant recipients (KTR), coming for routine check-ups to the transplant outpatient clinic Charité-Mitte, Berlin, Germany. Patients with active infections, malignancy, and acute rejection, a recent cardiovascular event at the time of collecting samples or unwilling to participate were excluded from this observational cohort. Local Ethics Committees approved this study, and informed consent was obtained from all study participants. Serum endostatin was measured using a sandwich enzyme immunoassay (Human Endostatin ELISA, cat. no. BI-20742, Biomedica, Austria) according to the instructions of the manufacturer. The limit of detection of the kit was $4 \mathrm{pmol} / \mathrm{L}$, and the average intra- and inter-assay coefficients of variation were $\leq 6$ and $5 \%$ respectively (described in detail on https://www.bmgrp.com/wp-content/ uploads/2019/01/BI-20742-Endostatin-US-ED-190402.pdf).

Blood and urine samples were collected from patients during routine visits from April 2012 until December 2012. Samples were kept frozen at $-80^{\circ} \mathrm{C}$ until use. Baseline data, that is, post-transplant duration, cold ischemia time, ages, genders of donor and recipient, panel reactive antibodies and human leukocyte antigen mismatches, and transplant survival were collected from patients' records. Baseline clinical parameters such as serum albumin, creatinine, total cholesterol, fasting blood glucose, calcium, phosphorus, as well as urinary protein excretion were all analyzed in the central clinical laboratories of Charité. Patients were followed for graft loss and all-cause mortality during the follow-up of 48 months. Graft loss was defined as return to dialysis, graft removal, re-transplantation, based on the judgment of the treating physicians. $\mathrm{Pa}$ tients' death with a functioning graft was considered death.

Descriptive statistics is expressed as medians (interquartile ranges) or numbers (\%). Optimal cutoff values were calculated as described. We analyzed the median and interquartile ranges for baseline characteristics. Comparisons were assessed by MannWhitney U test or Kruskal-Wallis test, as appropriate. Graft survival and time-to-event analysis was estimated by the KaplanMeier and differences were evaluated with a stratified log-rank test. Univariate analysis was used to determine the association between the endostatin concentration and graft survival. Multivariate Cox regression analysis was used to estimate the simultaneous effects of established and emerging graft risk factors on graft survival. Model A was adjusted for age, gender, and estimated glomerular filtration rate (eGFR), model $B$ was adjusted for same factors as model A, but endostatin and eGFR were analyzed as binary variables based on receiver operating characteristics (ROC) analysis for graft loss. Model C was adjusted for post-transplantation, optimal cut-off eGFR, serum phosphorus, and urinary protein excretion. $p$ value $<0.05$ was regarded statistically significant. All analyses were performed using SPSS version 25.0 (Chicago, IL, USA).

\section{Results}

\section{Participants}

A total of 574 stable KTRs were included in the study. Patients were followed for graft loss and all-cause mortality; 
Table 1. Baseline characteristics of patients by endostatin concentration

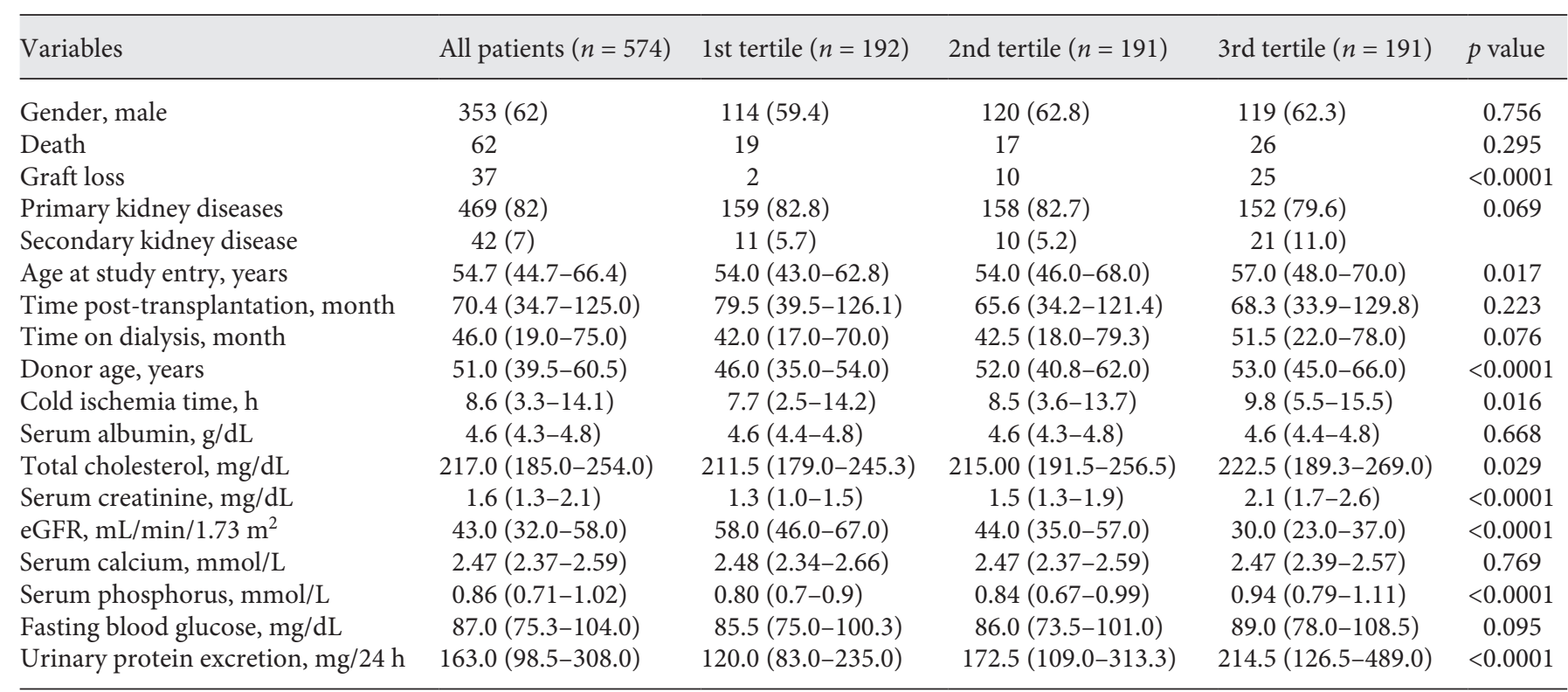

Data are given as median (interquartile ranges) or $n(\%)$.

$p$ values were calculated by Kruskal-Wallis $H$ test. Tertiles of endostatin: 1st: 39.60-120.07 (pmol/L); 2nd: 120.08-168.06 (pmol/L); 3rd:168.07-465.03 (pmol/L).

eGFR, estimated glomerular filtration rate.

during the 48 months follow-up period, 37 patients (6\%) had graft loss and 62 patients (11\%) died.

\section{Descriptive Data}

Table 1 presents the baseline demographic and clinical data of all patients. Baseline serum endostatin concentrations ranged from 39.60 to $465.03 \mathrm{pmol} / \mathrm{L}$ (median $142.83 \mathrm{pmol} / \mathrm{L}$ ). The distribution of baseline endostatin in the study population is shown in Figure 1. We divided our KTRs into tertiles according to endostatin concentrations (1st: 39.60-120.07; 2nd: 120.08-168.06; 3rd: 168.07-465.03). Patients in the 3rd tertile of endostatin were older, had older graft donor and longer cold ischemia time. In addition, serum creatinine, total cholesterol, phosphorus and urinary protein excretion were higher in these recipients. Median serum endostatin was higher in the graft loss group compared to that in the functioning graft group (207.72 [162.03-303.87] vs. 138.88 [106.81-178.55], $p<0.001$; online suppl. Table 1; for all online suppl. material, see www.karger.com/ doi/10.1159/000507824, Fig. 2). Interestingly, the median (interquartile range) of endostatin was significantly lower in Tacrolimus-treated recipients compared to CyA-treated recipients (127.99 [104.08-165.43] vs.

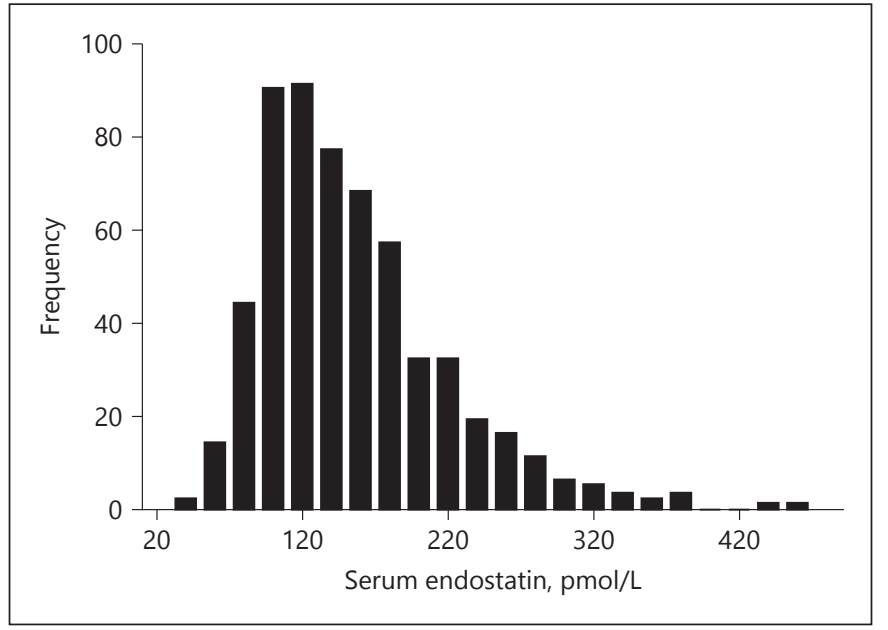

Fig. 1. Distribution of endostatin concentrations in the cohort. $n$ $=574$, mean $=154.29 \mathrm{pmol} / \mathrm{L} ; \mathrm{SD}$ 63.09; median (interquartile ranges) 142.83 (108.71-183.20) $\mathrm{pmol} / \mathrm{L}$.

154.31 [117.51-202.07], $p=0.0003$ ), also significantly lower when compared to Everolimus-treated recipients; (127.99 [104.08-165.43] vs. 153.06 [109.31-210.71], $p=$ 0.04; online suppl. Fig. 1). 


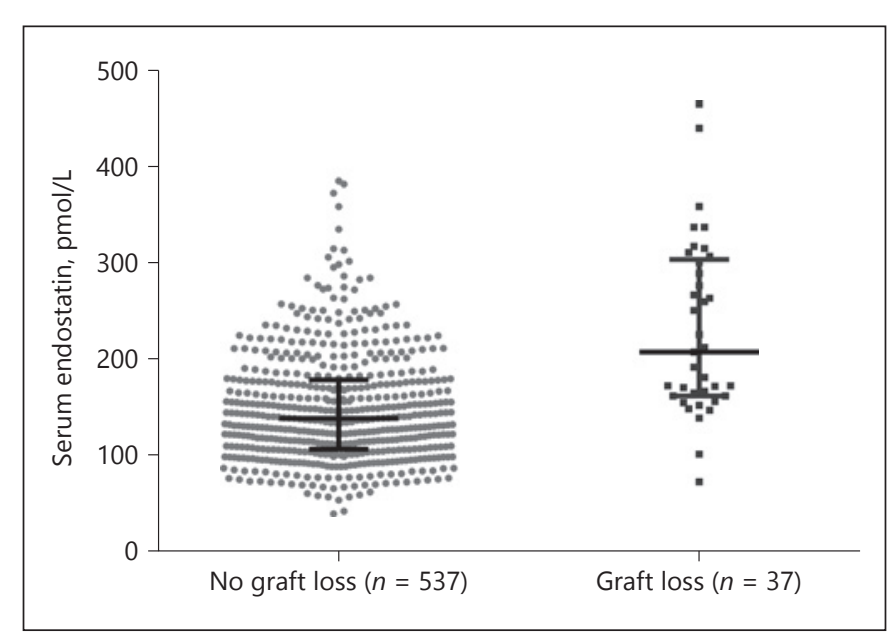

Fig. 2. Plots of serum endostatin concentrations. Median serum endostatin was significantly higher in the graft loss group compared to the functioning graft group using Mann-Whitney $\mathrm{U}$ test (207.72 [162.03-303.87] vs. 138.88 [106.81-178.55] pmol/L, $p<$ $0.001)$.

\section{Endostatin and Graft Loss}

Based on the ROC analysis, the optimal cutoff value of baseline serum endostatin for predicting graft loss was $147.3 \mathrm{pmol} / \mathrm{L}$ (area under the curve [AUC] 0.79, $p<0.001$; online suppl. Fig. 2). Compared to patients with endostatin $<147.3 \mathrm{pmol} / \mathrm{L}(n=307)$, patients with endostatin $\geq 147.3 \mathrm{pmol} / \mathrm{L}(n=267)$ had a nearly 15 -fold increase in the odds of for graft loss (OR 14.79, $p<0.001$ ). Similar statistical analyses were performed to test the optimal cutoff value of eGFR, serum phosphorus, and urinary protein excretion resulting in cutoffs of $52.5 \mathrm{~mL} / \mathrm{min} / 1.73 \mathrm{~m}^{2}$ (AUC $0.81, p<0.001$ ), $0.965 \mathrm{mmol} / \mathrm{L}$ (AUC 0.75, $p<0.001$ ), and $233.5 \mathrm{mg} / 24 \mathrm{~h}$ (AUC $0.79, p<0.001$ ) respectively.

Kaplan-Meier survival analysis indicated that patients in higher baseline endostatin concentrations groups had significantly lower graft survivals (log-rank test, $p<0.001$; Fig. 3).

A univariate Cox regression analysis (Table 2) indicated that higher endostatin concentrations $(\geq 147.3$ $\mathrm{pmol} / \mathrm{L}$ ) were positively correlated with an increased risk of graft loss (unadjusted hazard ratio [HR] 14.09; 95\% CI $4.33-45.89 ; p<0.001)$. As endostatin concentration was correlated with kidney function and age (online suppl. Fig. 3), to further address whether the effect of endostatin is independent of kidney function, age and other risk factors, we applied multiple regression models (Table 3). After adjusting for age, gender, and eGFR (Model A), circulating endostatin - treated as a continuous parameter multivariate Cox regression analysis showed an

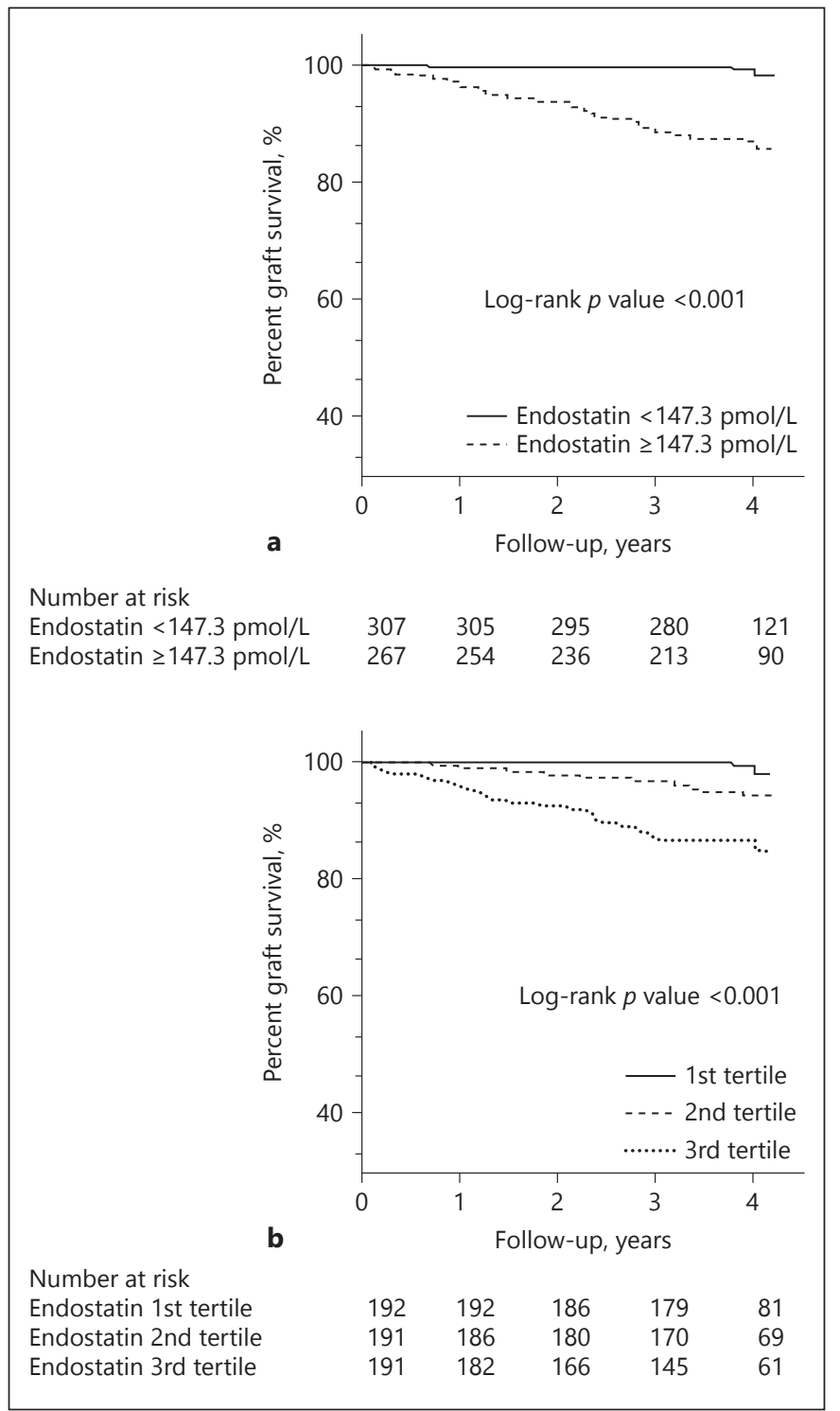

Fig. 3. Kaplan-Meier curves for graft loss. a Patients were grouped into 2 strands based on the optimal cut-off values of serum endostatin for graft loss. b Patients were grouped into 3 strands based on the tertile of endostatin.

independent association with graft loss (adjusted HR $1.01 ; 95 \%$ CI $1.00-1.01 ; p=0.002$ ). Using endostatin as a binary parameter based on ROC calculations of the optimal cut-off (Model B), endostatin was likewise found to be independently associated with graft loss (adjusted HR 14.19; 95\% CI 3.94-51.05; $p<0.001$ ). Additional adjustments for risk factors of graft loss, which showed significance in univariate Cox regression analysis (Model C), as post-transplantation time, eGFR $\leq 52.5 \mathrm{~mL} / \mathrm{min} / 1.73 \mathrm{~m}^{2}$, serum phosphorus $\geq 0.965 \mathrm{mmol} / \mathrm{L}$, as well as urinary 
Table 2. Univariate Cox regression analysis of factors predicting graft loss $(n=574)$

\begin{tabular}{|c|c|c|c|}
\hline Variable & Unadjusted HR & 95\% CI for HR & $p$ value \\
\hline Gender, male & 2.01 & $0.95-4.26$ & 0.07 \\
\hline Primary kidney diseases & 1.20 & $0.29-5.05$ & 0.80 \\
\hline Age at study entry, years & 1.00 & $1.00-1.00$ & 0.79 \\
\hline Time post-transplantation, month & 1.00 & $1.00-1.01$ & 0.03 \\
\hline Time on dialysis, month & 1.00 & $0.98-1.01$ & 0.34 \\
\hline Donor age, years & 1.01 & $0.98-1.03$ & 0.61 \\
\hline Cold ischemia time, $\mathrm{h}$ & 1.00 & $0.96-1.05$ & 0.91 \\
\hline PRA, \% & 0.99 & $0.95-1.04$ & 0.68 \\
\hline Serum albumin, g/dL & 0.61 & $0.21-1.78$ & 0.36 \\
\hline Total cholesterol, mg/dL & 1.00 & $0.99-1.01$ & 0.89 \\
\hline Serum creatinine, $\mathrm{mg} / \mathrm{dL}$ & 3.56 & $2.76-4.58$ & $<0.001$ \\
\hline $\mathrm{eGFR}, \mathrm{mL} / \mathrm{min} / 1.73 \mathrm{~m}^{2}$ & 0.91 & $0.88-0.94$ & $<0.001$ \\
\hline $\mathrm{eGFR} \leq 52.5\left(\mathrm{~mL} / \mathrm{min} / 1.73 \mathrm{~m}^{2}\right)$ & 3.02 & $1.17-7.81$ & 0.02 \\
\hline Serum calcium, $\mathrm{mmol} / \mathrm{L}$ & 0.30 & $0.06-1.50$ & 0.14 \\
\hline Serum phosphorus, $\mathrm{mmol} / \mathrm{L}$ & 12.27 & $5.16-29.20$ & $<0.001$ \\
\hline Serum phosphorus $(\geq 0.965 \mathrm{mmol} / \mathrm{L})$ & 5.10 & $2.55-10.21$ & $<0.001$ \\
\hline Serum endostatin, $\mathrm{pmol} / \mathrm{L}$ & 1.01 & $1.01-1.02$ & $<0.001$ \\
\hline Serum endostatin $(\geq 147.3 \mathrm{pmol} / \mathrm{L})$ & 14.09 & $4.33-45.89$ & $<0.001$ \\
\hline Fasting blood glucose, $\mathrm{mg} / \mathrm{dL}$ & 1.00 & $0.99-1.01$ & 0.80 \\
\hline Urinary protein excretion, $\mathrm{mg} / 24 \mathrm{~h}$ & 1.00 & $1.00-1.00$ & $<0.001$ \\
\hline Urinary protein excretion ( $\geq 233.5 \mathrm{mg} / 24 \mathrm{~h})$ & 8.12 & $3.32-19.89$ & $<0.001$ \\
\hline \multicolumn{4}{|l|}{ HLA mismatches } \\
\hline MMA_broad & 0.88 & $0.55-1.40$ & 0.58 \\
\hline MMB_broad & 1.16 & $0.75-1.80$ & 0.51 \\
\hline MMDR_broad & 0.97 & $0.60-1.54$ & 0.88 \\
\hline \multicolumn{4}{|l|}{ Medication } \\
\hline СyA & Reference & Reference & 0.64 \\
\hline Tacrolimus & 0.96 & $0.96(0.42-2.18)$ & 0.91 \\
\hline Everolimus & 1.70 & $1.70(0.65-4.47)$ & 0.28 \\
\hline Combined medication & 0.97 & $0.97(0.12-7.54)$ & 0.97 \\
\hline
\end{tabular}

Serum phosphorus, serum endostatin, urinary protein excretion was calculated both continuously and categorically respectively.

eGFR, estimated glomerular filtration rate; HR, hazard ratio; HLA, human leukocyte antigen; PRA, panel reactive antibodies.

protein excretion $\geq 233.5 \mathrm{mg} / 24 \mathrm{~h}$, revealed that higher endostatin concentration, remained both significantly and independently associated with graft loss (adjusted HR 8.34; 95\% CI 2.19-31.72; $p=0.002$ when using endostatin as a binary parameter).

\section{Endostatin and All-Cause Mortality}

In contrast to graft loss, baseline endostatin concentrations were not associated with all-cause mortality (online suppl. Table 2). The baseline median endostatin concentrations were $141.62(108.61-179.86) \mathrm{pmol} / \mathrm{L}$ in patients who died during follow-up and 146.90 (108.33-223.31) $\mathrm{pmol} / \mathrm{L}$ in patient who did not die, $p=0.22$. ROC curve analysis for all-cause mortality gave similar findings (AUC 0.55, $p=0.22$, online suppl. Fig. 4).

\section{Discussion}

Our study demonstrated that elevated baseline serum endostatin seems to be a promising biomarker predicting graft loss. The association between baseline endostatin concentrations and graft loss is stable, robust, and strong. This study confirms earlier findings that endostatin concentration was correlated with kidney function and age [24-27]. To further address whether the effect of endostatin is independent of kidney function, age and other risk factors, we applied multiple regression models (Table 3 ), all models demonstrated the same: endostatin is an independent risk of graft loss after kidney transplantation. This association between endostatin and graft loss seems to be even stronger as the established classical risk 
Table 3. Multivariate Cox regression analysis for graft loss

\begin{tabular}{|c|c|c|}
\hline & $\operatorname{HR}(95 \% \mathrm{CI})$ & $p$ value \\
\hline \multicolumn{3}{|l|}{ Model A } \\
\hline Endostatin, pmol/L & $1.01(1.00-1.01)$ & 0.002 \\
\hline Age, years & $1.00(1.00-1.00)$ & 0.10 \\
\hline Gender, male & $2.35(1.10-5.06)$ & 0.03 \\
\hline eGFR & $0.93(0.90-0.97)$ & $<0.001$ \\
\hline \multicolumn{3}{|l|}{ Model B } \\
\hline Endostatin $(\geq 147.3 \mathrm{pmol} / \mathrm{L})$ & $14.19(3.94-51.05)$ & $<0.001$ \\
\hline Age, years & $1.00(1.00-1.00)$ & 0.39 \\
\hline Gender, male & $1.95(0.91-4.20)$ & 0.09 \\
\hline $\mathrm{eGFR}\left(\leq 52.5 \mathrm{~mL} / \mathrm{min} / 1.73 \mathrm{~m}^{2}\right)$ & $1.05(0.37-2.99)$ & 0.92 \\
\hline \multicolumn{3}{|l|}{ Model C } \\
\hline Endostatin $(\geq 147.3 \mathrm{pmol} / \mathrm{L})$ & $8.34(2.19-31.72)$ & 0.002 \\
\hline Time post-transplantation, month & $1.00(1.00-1.01)$ & 0.91 \\
\hline $\operatorname{eGFR}\left(\leq 52.5 \mathrm{~mL} / \mathrm{min} / 1.73 \mathrm{~m}^{2}\right)$ & $1.91(0.65-5.63)$ & 0.24 \\
\hline \multicolumn{3}{|l|}{ Urinary protein excretion } \\
\hline$(\geq 233.5 \mathrm{mg} / 24 \mathrm{~h})$ & $5.44(2.20-13.46)$ & $<0.001$ \\
\hline Serum phosphorus $(\geq 0.965 \mathrm{mmol} / \mathrm{L})$ & $3.30(1.45-7.52)$ & 0.005 \\
\hline
\end{tabular}

In model A, circulating endostatin levels were treated as a continuous parameter. In models B and C, endostatin levels were included into the model as categorical variable.

eGFR, estimated glomerular filtration rate; HR, hazard ratio.

factors for graft loss: decreased glomerular filtration rate (GFR) and increased urinary protein excretion.

A recent clinical study showed that the median plasma endostatin concentrations and interquartile ranges in CKD patients were significantly higher compared to controls without CKD (265.8 pmol/L, 189.6-363.3 vs. 119.1 pmol/L, 102.7-136.1; $p<0.001)$. Endostatin concentrations were correlated with the severity of CKD, the occurrence of cardiovascular events and mortality in a doseresponse manner [24]. In patients with type 2 diabetes, elevated levels of endostatin were associated with increased risk of diabetic nephropathy [28].

According to the instructions of the manufacturer, the median value of serum endostatin from apparently healthy individuals $(n=59)$ is $51 \mathrm{pmol} / \mathrm{L}$ (https://www. b mgrp.com/wp-content/uploads/2019/01/BI20742-Endostatin-US-ED-190402.pdf). The endostatin concentrations observed in our study are about 3 times higher than those in the general population with normal GFR; however, they were in good agreement with above measurements in CKD patients. We are of the opinion that endostatin is a $20 \mathrm{kD}$ peptide, which is freely filtered by the glomeruli. Thus, its blood concentrations are affected by an impairment of GFR as it was shown for other peptides of similar size such as troponins [29].
Animal studies suggest that endostatin might not just be a biomarker of kidney function rather than being a yet unknown novel player. Endostatin transgenic mice develop renal interstitial fibrosis. Similar findings were obtained when chronically giving endostatin via minipumps [21]. These findings were thus in good agreement with clinical studies showing that higher circulating endostatin levels are associated with lower GFR and higher levels of albuminuria [26].

Two small clinical studies analyzed the association of endostatin in KTRs, but neither of them focused on graft survival. One study demonstrated that endostatin levels were higher in KTRs compared with liver transplant recipients and healthy controls, and were associated with the development of atherosclerosis after kidney transplantation [30]. Another study found a significant negative correlation between GFR, pulse wave velocity, and endostatin concentrations in KTRs [31]. Our study is the first to report an independent association between endostatin and graft survival in KTRs.

The rate of acute graft loss after kidney transplantation decreased significantly in the past decades [6]. Chronic graft dysfunction characterized by the development of glomerulosclerosis, tubular interstitial fibrosis and atrophy is nowadays the major clinical challenge $[32,33]$.

Endostatin is known as a powerful angiogenesis inhibitor, and is released from collagen XVIII, which is a component of subendothelial and subepithelial basement membranes especially in vessels with fenestrated endothelium, like renal glomeruli and peritubular capillaries $[34,35]$. Elevated blood concentrations of endostatin were seen under conditions of vascular endothelial dysfunction and extracellular matrix remodeling [23].

Angiogenesis is the proliferation of endothelial and smooth muscle cells to form new blood vessels from preexisting vessels. Angiogenesis is not only involved in pathologic situations such as tumor growth and metastasis, but also in therapeutic angiogenesis. It is essential for tissue regeneration after ischemic damage and organ development $[15-17,36]$. A study demonstrated that direct transplantation of bone marrow-derived endothelial progenitor cells (BM-derived EPCs) into ischemic hearts induces significant and sustained increase in various angiogenic, antiapoptotic, and chemoattractant factors during the critical period of infarct repair and remodeling [17]. One latest study reported that angiogenesis contributes to the regeneration of a transplanted liver in vivo [15], and thus reinforcing the impact of angiogenesis in preservation of organ function of the transplanted liver. However, this was not investigated after kidney transplantation so far. 
Microvascular rarefaction is a common process observed during fibrotic remodeling seen in all organs. With respect to kidney function, it was shown that loss of glomerular function is associated with the presence of tubulointerstitial fibrosis, which is characterized by a substantial rarefaction of peritubular capillaries. Dysregulated expression of endogenous angiogenic inhibitors such endostatin might influence the balance between capillary formation and regression, resulting in capillary rarefaction $[18-20,25,37]$.

So far, there are some studies proving evidence for a correlation between the endostatin levels and vascular changes. In vitro studies showed that endostatin blocks the proliferation and organization of endothelial cells into new blood vessel [38]. In animal studies, elevated endostatin levels have shown to be correlated with increased microvascular rarefaction/interstitial fibrosis in aging mice [21, 39]. In patients with systemic sclerosis, elevated serum endostatin levels are associated with digital vascular damage [40].

Taking into account the published data on endostatin $[18-21,39]$ and our own findings, we propose the following hypothesis: chronic graft failure is characterized by fibrosis of the transplanted kidney. In the course of fibrotic remodeling, there might also be an increased synthesis and later also breakdown of collagen XVIII and thus an increased release of endostatin. This endostatin resulting from fibrotic organ remodeling might influence the balance between capillary formation and regression in a negative way because endostatin is a known antiangiogenic factor, resulting in capillary rarefaction. The endostatin-mediated decrease in capillary density would worsen the blood and oxygen supply to the transplanted kidney and thus contribute to further organ failure.

Our study consists of a well-defined cohort of KTRs without loss of follow-up, and is the first one to present the independent association between circulating endostatin concentrations and graft loss. Three independent statistic methods (ROC analysis, Kaplan-Meier analysis, and Cox proportional-hazards model) were performed, and all results consistently showing that elevated endostatin concentration was independently and strongly associated with graft survival. We also acknowledge several limitations of this study. First, our study was a single center study and the number of graft loss events is relatively small and further independent replication studies are necessary. Second, the endostatin concentrations were measured only once at study entry, hence it is impossible to investigate the alteration in endostatin levels over time in this cohort. Furthermore, systematic biopsies were not done in our study population, so we cannot correlate capillary density in the biopsies to endostatin concentrations, and this cross-sectional study can only show the association between baseline endostatin and graft survival and further research is needed to prove causality. Moreover, our cohort was not followed for late acute rejections. Although considering the previous study [10], we thus would have expected only very few cases of acute rejections in our stable transplant cohort that might have no significant effect on our findings.

In conclusion, increased serum endostatin concentrations at baseline are independently associated with the risk of graft loss in prevalent KTRs. Its association with graft loss seems to be even stronger as compared the established classical risk factors for graft loss: decreased GFR and increased urinary protein excretion (Table 3).

\section{Acknowledgments}

Biomedica Medizinprodukte GmbH, A-1210 Wien, Divischgasse 4, Austria, provided the Human Endostatin ELISA (cat. no. BI-20742). China Scholarship Council supported C.C., S.Z., and Y.X.

\section{Statement of Ethics}

Local Ethics Committees approved this study, and informed consent was obtained from all study participants.

\section{Disclosure Statement}

The authors have no conflicts of interest to declare.

\section{Funding Sources}

The authors declare no funding was received for this study.

\section{Author Contributions}

B.H.: research idea and study design. C.C. and B.H.: data acquisition/analysis. C.C., A.A.H., and S.Z. statistical analysis. All authors: data interpretation. B.H., B.K.K., and S.E. supervision or mentorship. Each author contributed important intellectual content during manuscript drafting or revision and accepts accountability for the overall work by ensuring that questions pertaining to the accuracy or integrity of any portion of the work are appropriately investigated and resolved. 


\section{References}

1 Xie Y, Bowe B, Mokdad AH, Xian H, Yan Y, Li T, et al. Analysis of the Global Burden of Disease study highlights the global, regional, and national trends of chronic kidney disease epidemiology from 1990 to 2016. Kidney Int. 2018 Sep;94(3):567-81.

2 Port FK, Wolfe RA, Mauger EA, Berling DP, Jiang K. Comparison of survival probabilities for dialysis patients vs cadaveric renal transplant recipients. JAMA. 1993 Sep;270(11): 1339-43.

3 Ojo AO, Port FK, Wolfe RA, Mauger EA, Williams L, Berling DP. Comparative mortality risks of chronic dialysis and cadaveric transplantation in black end-stage renal disease patients. Am J Kidney Dis. 1994 Jul;24(1):59-64.

4 Schnuelle P, Lorenz D, Trede M, Van Der Woude FJ. Impact of renal cadaveric transplantation on survival in end-stage renal failure: evidence for reduced mortality risk compared with hemodialysis during long-term follow-up. J Am Soc Nephrol. 1998 Nov; 9(11):2135-41.

5 Outcomes. KDIG. KDIGO clinical practice guideline on the evaluation and management of candidates for kidney transplantation (Draft). 2018.

6 Hariharan S, Johnson CP, Bresnahan BA, Taranto SE, McIntosh MJ, Stablein D. Improved graft survival after renal transplantation in the United States, 1988 to 1996. N Engl J Med. 2000 Mar;342(9):605-12.

7 Meier-Kriesche HU, Schold JD, Srinivas TR, Kaplan B. Lack of improvement in renal allograft survival despite a marked decrease in acute rejection rates over the most recent era. Am J Transplant. 2004 Mar;4(3):378-83.

8 Thiel G, Bock A, Spöndlin M, Brunner FP, Mihatsch M, Rufli T, et al. Long-term benefits and risks of cyclosporin A (sandimmun) - an analysis at 10 years. Transplant Proc. 1994 Oct;26(5):2493-8.

9 Almond PS, Matas A, Gillingham K, Dunn DL, Payne WD, Gores P, et al. Risk factors for chronic rejection in renal allograft recipients. Transplantation. 1993 Apr;55(4):752-6.

10 Basadonna GP, Matas AJ, Gillingham KJ, Payne WD, Dunn DL, Sutherland DE, et al. Early versus late acute renal allograft rejection: impact on chronic rejection. Transplantation. 1993 May;55(5):993-5.

11 Escofet X, Osman H, Griffiths DF, Woydag S, Adam Jurewicz W. The presence of glomerular sclerosis at time zero has a significant impact on function after cadaveric renal transplantation. Transplantation. 2003 Feb;75(3): 344-6.

$12 \mathrm{Li} \mathrm{C}$, Yang CW. The pathogenesis and treatment of chronic allograft nephropathy. Nat Rev Nephrol. 2009 Sep;5(9):513-9.

13 O'Reilly MS, Boehm T, Shing Y, Fukai N, Vasios G, Lane WS, et al. Endostatin: an endog- enous inhibitor of angiogenesis and tumor growth. Cell. 1997 Jan;88(2):277-85.

14 Ding YH, Javaherian K, Lo KM, Chopra R, Boehm T, Lanciotti J, et al. Zinc-dependent dimers observed in crystals of human endostatin. Proc Natl Acad Sci USA. 1998 Sep; 95(18):10443-8.

15 Jung DH, Moon SH, Park SJ, Kim EJ, Jang IH, Park CS, et al. Angiogenesis in the Transplanted Donor Graft after Living-Donor Liver Transplantation. Transplantation. 2018 Jan;102(1):e26-9.

16 Uda Y, Hirano T, Son G, Iimuro Y, Uyama N, Yamanaka J, et al. Angiogenesis is crucial for liver regeneration after partial hepatectomy. Surgery. 2013 Jan;153(1):70-7.

17 Cho HJ, Lee N, Lee JY, Choi YJ, Ii M, Wecker $\mathrm{A}$, et al. Role of host tissues for sustained humoral effects after endothelial progenitor cell transplantation into the ischemic heart. J Exp Med. 2007 Dec;204(13):3257-69.

18 Tanaka T, Nangaku M. Angiogenesis and hypoxia in the kidney. Nat Rev Nephrol. 2013 Apr;9(4):211-22.

19 Maeshima Y, Makino H. Angiogenesis and chronic kidney disease. Fibrogenesis Tissue Repair. 2010 Aug;3(1):13.

20 Lerman LO, Chade AR. Angiogenesis in the kidney: a new therapeutic target? Curr Opin Nephrol Hypertens. 2009 Mar;18(2):160-5.

21 Lin CH, Chen J, Zhang Z, Johnson GV, Cooper AJ, Feola J, et al. Endostatin and transglutaminase 2 are involved in fibrosis of the aging kidney. Kidney Int. 2016 Jun;89(6):1281-92.

22 Carlsson AC, Östgren CJ, Länne T, Larsson A, Nystrom FH, Ärnlöv J. The association between endostatin and kidney disease and mortality in patients with type 2 diabetes. $\mathrm{Di}$ abetes Metab. 2016 Nov;42(5):351-7.

23 Carlsson AC, Ruge T, Sundström J, Ingelsson E, Larsson A, Lind L, et al. Association between circulating endostatin, hypertension duration, and hypertensive target-organ damage. Hypertension. 2013 Dec;62(6): 1146-51.

24 Chen J, Hamm LL, Kleinpeter MA, Husserl F, Khan IE, Chen CS, et al. Elevated plasma levels of endostatin are associated with chronic kidney disease. Am J Nephrol. 2012;35(4): 335-40.

25 Kato Y, Furusyo N, Tanaka Y, Yamasaki S, Ueyama T, Takayama K, et al. Association of the Serum Endostatin Level, Renal Function, and Carotid Atherosclerosis of Healthy Residents of Japan: Results from the Kyushu and Okinawa Population Study (KOPS). J Atheroscler Thromb. 2018 Sep;25(9):829-35.

26 Ruge T, Carlsson AC, Larsson TE, Carrero JJ, Larsson A, Lind L, et al. Endostatin level is associated with kidney injury in the elderly: findings from two community-based cohorts. Am J Nephrol. 2014;40(5):417-24.
27 Kanbay M, Afsar B, Siriopol D, Unal HU, Karaman M, Saglam M, et al. Endostatin in chronic kidney disease: associations with inflammation, vascular abnormalities, cardiovascular events and survival. Eur J Intern Med. 2016 Sep;33:81-7.

28 Chauhan K, Verghese DA, Rao V, Chan L, Parikh CR, Coca SG, et al. Plasma endostatin predicts kidney outcomes in patients with type 2 diabetes. Kidney Int. 2019 Feb;95(2): $439-46$.

29 Wiessner R, Hannemann-Pohl K, Ziebig R, Grubitzsch H, Hocher B, Vargas-Hein O, et al. Impact of kidney function on plasma troponin concentrations after coronary artery bypass grafting. Nephrol Dial Transplant. 2008 Jan;23(1):231-8.

30 Iqbal CW, Dean PG, Ishitani MB. Early-onset coronary artery disease after pediatric kidney transplantation: implicating the angiogenesis inhibitor, endostatin. Am Surg. 2011 Jun; 77(6):731-5.

31 Gurlek Demirci B, Sezer S, Uyanik Yildirim S, Kaynar Erdoğan M, Tutal E, Özdemir Ö, et al. FGF23, NGAL, and Endostatin: the Predictors of Allograft Function in Renal Transplant Recipients. Exp Clin Transplant. 2018; 16(Suppl 1):136-9.

32 Nankivell BJ, Chapman JR. Chronic allograft nephropathy: current concepts and future directions. Transplantation. 2006 Mar;81(5): 643-54.

33 Chapman JR, O'Connell PJ, Nankivell BJ. Chronic renal allograft dysfunction. J Am Soc Nephrol. 2005 Oct;16(10):3015-26.

34 Seppinen L, Pihlajaniemi T. The multiple functions of collagen XVIII in development and disease. Matrix Biol. 2011 Mar;30(2):8392.

35 Ferreras M, Felbor U, Lenhard T, Olsen BR, Delaissé J. Generation and degradation of human endostatin proteins by various proteinases. FEBS Lett. 2000 Dec;486(3):24751.

36 Folkman J. The role of angiogenesis in tumor growth. Semin Cancer Biol. 1992 Apr;3(2) 65-71.

37 Lähteenvuo J, Rosenzweig A. Effects of aging on angiogenesis. Circ Res. 2012 Apr;110(9): 1252-64.

38 Folkman J, Kalluri R. Cancer without disease. Nature. 2004 Feb;427(6977):787.

39 Lin CH, Chen I, Ziman B, Marshall S, Maizel J, Goligorsky MS. Endostatin and kidney fibrosis in aging: a case for antagonistic pleiotropy? Am J Physiol Heart Circ Physiol. 2014 Jun;306(12):H1692-9.

40 Gigante A, Navarini L, Margiotta D, Barbano B, Afeltra A, Rosato E. In systemic sclerosis, microvascular and hands digital arteries damage correlates with serum levels of endostatin. Microcirculation. 2018 May;25(4):e12449. 